\title{
Continuous-flow Cell Cycle Fractionation of Eukaryotic Micro-organisms
}

\author{
By D. LLOYD, LYNDA JOHN, MERRYL HAMILL, CAROL PHILLIPS, \\ J. KADER AND S. W. EDWARDS \\ Department of Microbiology, University College, Newport Road, Cardiff CF2 ITA
}

(Received 12 August 1976; revised 25 September 1976)

INTRODUCTION

Previous methods of cell cycle fractionation have been based on sedimentation of organisms through density gradients in tubes or zonal centrifuge rotors (Warmsley \& Pasternak, 1970). Both rate-sedimentation and equilibrium density methods have been used, for example for Saccharomyces cerevisiae and for Schizosaccharomyces pombe (Sebastian, Carter \& Halvorson, 197I ; Poole \& Lloyd, I973; Poole, Lloyd \& Chance, 1974; Edwards \& Lloyd, 1977). However, these methods suffer from a major drawback. Organisms must be harvested from their growth media, resuspended, loaded on to the gradient, then separated and recovered. Such procedures may take up to $I \mathrm{~h}$ during which time the organisms are exposed to conditions of nutrient and oxygen deprivation, suboptimal growth temperatures and, in some cases, unfavourable osmotic environments. Continuous-flow cell cycle fractionation in a Sharples Supercentrifuge is completed in less than $15 \mathrm{~min}$ (for a Io 1 culture), and thus obviates the unfavourable conditions inherent in gradient methods; size selection is just as efficient.

\section{METHODS}

Organisms. The methods used for the maintenance and growth of organisms have been described for Schizosaccharomyces pombe 972h- (Poole, Lloyd \& Kemp, 1973), Candida utilis NCYCI93 (Light \& Garland, 1971), Saccharomyces cerevisiae D22a (Deutsch et al., 1974), Acanthamoeba castellanii (Griffiths \& Chagla, 1972), Crithidia fasciculata (Edwards \& Lloyd, 1973) and Tetrahymena pyriformis strain ST (Lloyd et al., I97I). Carbon sources in the yeast cultures were glucose, acetate and glycerol for $S c h i z$. pombe, $C$. utilis and $S$. cerevisiae respectively.

Coulter counter analysis. The frequency distribution of particle volumes was determined using a model $Z_{B}$ Coulter counter (Coulter Electronics, Dunstable, Bedfordshire) together with a Coulter Channelyzer and XY recorder II. Samples were diluted in Isoton (Coulter Electronics), except, for $A$. castellanii and $T$. pyriformis, filtered growth medium was used. Particle counts were made immediately using a $70 \mu \mathrm{m}$ orifice (except, for $T$. pyriformis, the orifice diameter was $140 \mu \mathrm{m}$ ). Frequency distributions of particle volumes were accumulated in the Channelyzer over arbitrary periods of sample analysis until 2048 particles had been counted in the peak channel; they were then plotted out on the recorder. Total particle counts were made on $0.5 \mathrm{ml}$ samples; dilutions were such that the number of particles did not exceed $4 \times 10^{4}$ in this sample volume. Settings were $\mathrm{I} \times \mathrm{A}=\mathrm{I} \cdot 0^{\circ}$ (for $S$ chiz. pombe, $S$. cerevisiae and $C r$. fasciculata), $\mathrm{I} \times \mathrm{A}=0.5$ (for $C$. utilis), $\mathrm{I} \times \mathrm{A}=\mathrm{I} 6$ (for A. castellanii) and $\mathbf{I} \times \mathbf{A}=\mathbf{I} 6$ (for $T$. pyriformis); edit switch, on; upper threshold, off; lower threshold, Io; gain switch, 5 ; matching resistance, $20 \mathrm{k} \Omega$. 
Centrifugation. The rotor was accelerated to the desired speed and water was allowed to flow through the continuous-flow system which consisted of a feed nozzle, selected to provide a suitable calibrated flow rate, leading into the bottom of a BR7-34 separator bowl of a Sharples Supercentrifuge (IA, open type; Pennwalt, Doman Road, Camberley, Surrey). The rotor was fitted with a corrugated rubber liner with 18 grooves running around its circumference, inserted on a thin plastic liner for ease of insertion and removal. The liquid entered the bottom of the rotor coaxially, was distributed by quadrant plates, and then passed up the bowl wall over the corrugated liner before being discharged in an annular layer through the overflow weir. The depth of the liquid layer on the bowl wall was dictated by the difference between the wall radius $(R)$ and the radius of the overflow weir $(S)$; this depth was constant at rotor speeds between 10000 and 23000 rev. $\min ^{-1}$ and at flow rates between 0.3 and $\mathrm{x} \cdot 91 \mathrm{~min}^{-1}$.

Assuming that no re-entrainment of previously sedimented particles occurs as the liquid leaves the rotor and that the flow within the rotor is streamlined (Bradley, 1962), the particle trajectory within the system is the resultant of the radial settling velocity and the velocity distribution across the liquid layer. $D_{c}$, the size of particle which will just settle from the liquid in the time taken for the liquid to move the length of the bowl wall, is given by the equation:

$$
D_{\mathrm{c}}^{2}=\frac{Q}{\pi K L\left(R^{2}-S^{2}\right)} \frac{K_{1}}{2}\left[R^{2} \ln \left(\frac{R}{x_{0}}\right)-\frac{R^{2}-x_{0}^{2}}{2}-S^{2} \ln ^{2}\left(\frac{R}{x_{0}}\right)\right]
$$

in which $Q$ is the flow rate (in $\mathrm{cm}^{3} \mathrm{~s}^{-1}$ ), $L, R$ and $S$ are the length, outer and inner radii of the liquid column respectively (all in $\mathrm{cm}$ ), $x_{0}$ is the radius at entry (in $\mathrm{cm}$ ),

$$
K_{1}=\frac{R^{2}-S^{2}}{\frac{3}{4} S^{4}+\frac{1}{4} R^{4}-R^{2} S^{2}-S^{4} \ln S / R}\left(\text { in } \mathrm{cm}^{-2}\right) \text { and } K=\frac{(d-\rho) \omega^{2}}{\mathrm{I} 8 \eta}
$$

in which $d$ and $\rho$ are the densities of the particle and liquid respectively (in $\mathrm{g} \mathrm{cm}^{-3}$ ), $\omega$ is the angular velocity (in $\mathrm{rad} \mathrm{s}^{-1}$ ) and $\eta$ is the viscosity of the liquid (in $\mathrm{g} \mathrm{cm}^{-1} \mathrm{~s}^{-1}$ ).

After checking the flow rate and rotor speed (using a calibrated Variac control unit), the siphon was transferred from the water reservoir to the exponentially growing culture in the growth vessel. The culture was allowed to flow through the system until only ioo $\mathrm{ml}$ remained, when the flow was abruptly terminated by clamping the inlet tube with artery forceps. The rotor was run for another minute at the same rotor speed and then accelerated to top speed $\left(23000 \mathrm{rev}\right.$. $\left.\mathrm{min}^{-1}\right)$ for $5 \mathrm{~min}$ in order to pack the cells in the grooves of the insert.

After deceleration, the rotor was dismantled and the rubber liner was carefully removed on its plastic insert. The rubber liner was then unrolled, and organisms were recovered from the grooves using a spatula and Pasteur pipette. Successful separation of various size classes of organisms requires that the full length of the rotor is utilized for sedimentation; suitable conditions of flow rate and rotor speed may be calculated from equation (I) or by practical determination of the rotor speed which is necessary to prevent any organisms appearing in the effluent from the centrifuge at a fixed flow rate. We have found that at high flow rates (e.g. $1900 \mathrm{ml} \mathrm{min}^{-1}$ ) a considerable end-effect at the bottom of the rotor leads to mixing of cell sizes, and hence to loss of resolution in the first six fractions subsequently obtained. At rotor speeds lower than $8000 \mathrm{rev}$. $\mathrm{min}^{-1}$, the surface of the liquid column is no longer vertical and some loss of holding volume occurs. 


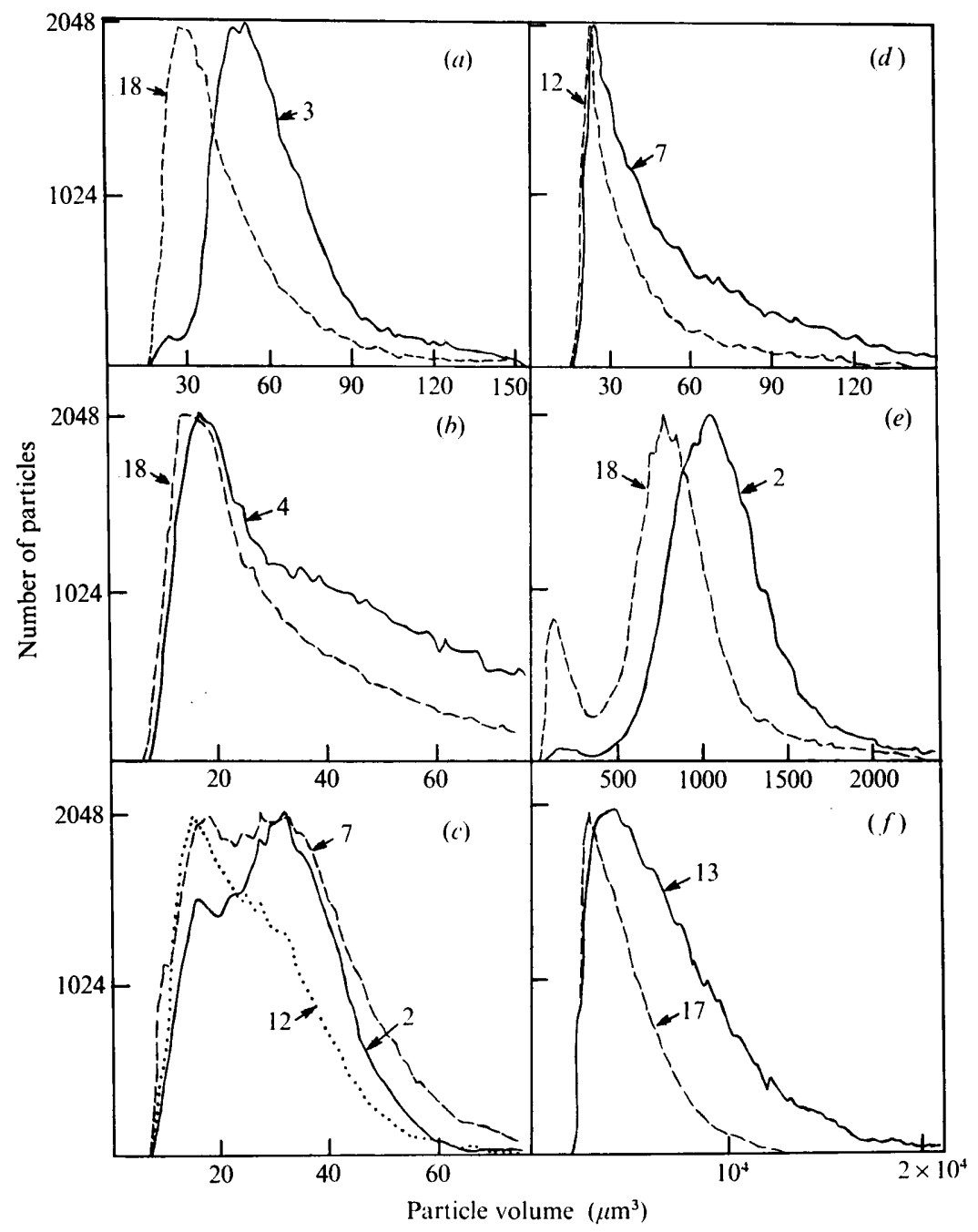

Fig. I. Size distributions of fractions from exponentially growing populations selected by continuous-flow cell cycle analysis. Measurements were made in a Coulter counter fitted with a Channelyzer and XY recorder. (a) Schizosaccharomyces pombe $972 \mathrm{~h}^{-}$, (b) Saccharomyces cerevisiae D22a, (c) Candida utilis NCYC193, (d) Crithidia fasciculata, (e) Acanthamoeba castellanii, $(f)$ Tetrahymena pyriformis ST. Numbers refer to fraction numbers indicated in Fig. 2.

\section{RESULTS AND DISCUSSION}

Representative frequency distribution histograms obtained from cell size analysis are shown in Fig. I for each of the organisms. Large organisms were predominantly sedimented near the feed inlet end of the rotor (low fraction numbers), while the smallest size classes sedimented near the outlet end. For $S$. pombe and the three protozoa, positively skewed distributions with a single mode were obtained; those for the budding yeasts were more complex, with $C$. utilis displaying a bimodal frequency distribution with an increasing proportion of the larger of the two populations in the higher fraction numbers. With Cr. fasciculata, rosette formation made a one-step fractionation procedure impossible; 


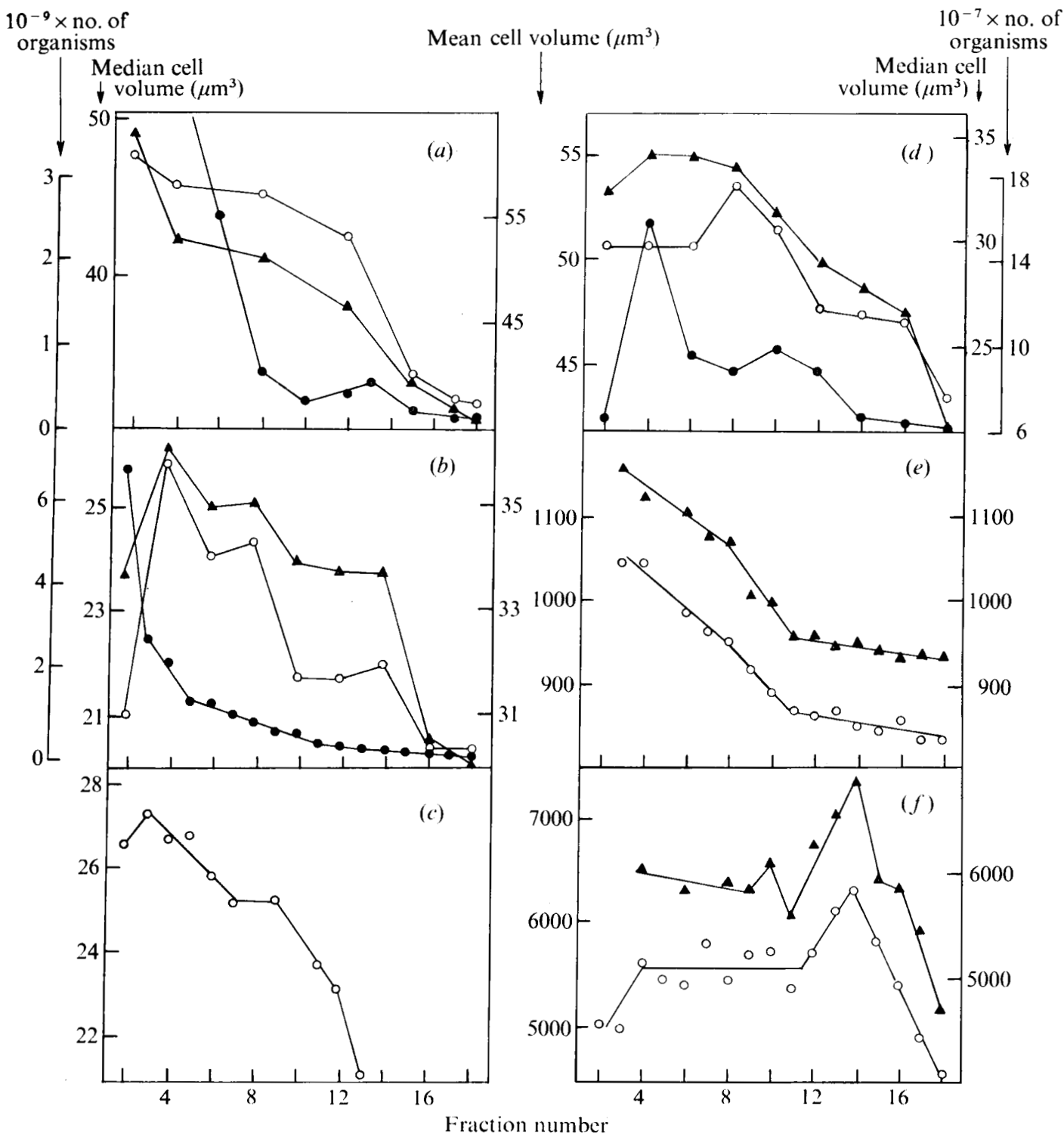

Fig. 2. Distributions of organisms along the length of the corrugated rubber insert after continuousflow cell cycle fraotionation. Organisms were grown in batch cultures to the mid-exponential phase of growth then 21 of culture was passed through the continuous-flow centrifuge. Recovered cells were subjected to Coulter size analysis: total cell numbers $(\Theta)$, median cell volumes $(O)$, mean cell volumes (A). (a) Schizosaccharomyces pombe $972 \mathrm{~h}^{-}$(10000 rev. $\left.\mathrm{min}^{-1}, 750 \mathrm{ml} \mathrm{min}^{-1}\right) ;(b)$ Saccharomyces cerevisiae D22a (I $800 \mathrm{rev} . \mathrm{min}^{-1}, 600 \mathrm{ml} \mathrm{min}-1$ ); (c) Candida utilis NCYCI93

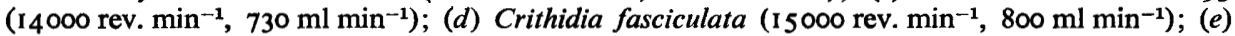

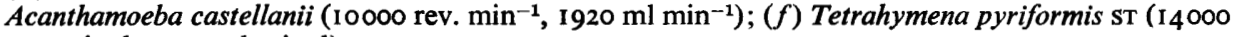
rev. $\left.\min ^{-1}, 1700 \mathrm{ml} \mathrm{min}^{-1}\right)$. 
organisms were therefore harvested by centrifugation in the $6 \times 11$ rotor of an MSE Mistral centrifuge at $3000 \mathrm{rev}$. $\mathrm{min}^{-1}$ for $10 \mathrm{~min}$ and resuspended in distilled water (volume equal to that of the original culture) before continuous-flow cell cycle fractionation.

Figure 2 shows median and mean cell volumes as a function of the path length (fraction number) for sedimentation within the rotor. For all six organisms there was effective resolution of subpopulations of differing size distribution, although some anomalous values were found for the first few fractions. This effect resulted from turbulence near the feed inlet and was particularly marked at high flow rates (e.g. T. pyriformis, Fig. $2 f$ ). Microscopical examination of the rotor effluents confirmed that under the centrifugation conditions used, all cells were retained within the rotor in the six experiments shown.

The present method of cell cycle analysis has been developed from the continuous-flow size selection procedure for the preparation of synchronous cultures (Lloyd et al., 1975), and has all the advantages of that method, in that it is technically simple and rapid, and avoids the prior harvesting of organisms and their exposure to density gradients. It can be applied to any type of culture in which organisms do not grow in chains or clusters and in which no flocculation occurs. It is applicable to mechanically fragile organisms, such as amoebae, and to highly motile organisms, such as $C r$. fasciculata and $T$. pyriformis; it might also find application in studies of cells of higher organisms grown in liquid culture.

We thank Pennwalt Ltd for their generosity in lending a Sharples Supercentrifuge and providing technical information. During the course of this work S.W.E. was in receipt of a studentship from the Medical Research Council.

\section{REFERENCES}

Bradley, D. (1962). Centrifugal methods of size analysis of sub-micron particles (part 2). Chemical and Process Engineering 43, 634-637.

Deutsch, J., Dujon, B., Netter, P., Petrochmo, E., Slonimski, P. P., Bolotin-Fukuhara, M. \& Coen, D. (1974). Inter-relationships between loss of the $\rho^{+}$factor and the loss of the drug resistance mitochondrial genetic markers. Genetics 76, 195-219.

EdwARDS, C. \& LLOYD, D. (1973). Terminal oxidases and carbon monoxide-reacting haemoproteins in the trypanosomatid, Crithidia fasciculata. Journal of General Microbiology 79, 275-284.

EDWARDS, S. W. \& LLOYD, D. (1977). Mitochondrial adenosine triphosphatase of the fission yeast, Schizosaccharomyces pombe $972 \mathrm{~h}^{-}$; changes in activity and oligomycin sensitivity during the cell cycle. Biochemical Journal 162, 39-46.

Grifriths, A. J. \& ChAGLA, A. H. (1972). The growth and encystment of Acanthamoeba castellanii. Journal of Protozoology Sig, Al 30 .

Light, P. A. \& GaRLAND, P. B. (1971). A comparison of mitochondria from Torulopsis utilis grown in continuous culture with glycerol, iron, ammonium, magnesium or phosphate as the growth-limiting nutrients. Biochemical Journal 124, I 23-1 34.

Lloyd, D., Brightwell, R., Venables, S. E., ROACh, G. I. \& TURNER, G. (197I). Subcellular fractionation of Tetrahymena pyriformis ST by zonal centrifugation: changes in activities and distribution of enzymes during the growth cycle and on starvation. Journal of General Microbiology 65, 209-223.

LLOYD, D., JohN, L., EDWARDS, C. \& CHAGLA, A. H. (I975). Synchronous cultures of micro-organisms: large-scale preparation by continuous-flow size selection. Journal of General Microbiology 88, I 53-1 58.

Poole, R. K. \& LLOYD, D. (1973). Oscillations of enzyme activities during the cell cycle of a glucoserepressed fission yeast, Schizosaccharomyces pombe. Biochemical Journal 136, 195-207.

Poole, R. K., Lloyd, D. \& CHANCE, B. (1974). The development of cytochromes during the cell cycle of a glucose-repressed fission yeast Schizosaccharomyces pombe. Biochemical Journal 138, $201-210$.

POOLE, R. K., LLOYD, D. \& KEMP, R. B. (1973). Respiratory oscillations and heat evolution in synchronously dividing cultures of the fission yeast, Schizosaccharomyces pombe. Journal of General Microbiology 72, 209-220.

Sebastian, J., Carter, B. L. A. \& Halvorson, H. O. (197I). Use of yeast populations fractionated by zonal centrifugation to study the cell cycle. Journal of Bacteriology 108, 1045-1050.

WARMSLeY, A. M. H. \& PASTERNAK, C. A. (1970). The use of conventional and zonal centrifugation to study the life cycle of mammalian cells. Biochemical Journal 119, 493-499. 